\title{
A Remark on a Standard and Linear Vector Network Equilibrium Problem with Capacity Constraints $^{1}$
}

\author{
S. J. $\mathrm{LI}^{2}$ \\ College of Mathematics and Science, \\ Chongqing University, Chongqing, 400044, China. \\ E-mail: lisj@cqu.edu.cn \\ K. L. TEO \\ Department of Mathematics and Statistics, Curtin University of Technology \\ GPO Box U1987, Perth, W. A. 6845, Australia \\ E-mail: K.L.Teo@curtin.edu.au \\ X. Q. YANG \\ Department of Applied Mathematics, The Hong Kong Polytechnic University, \\ Kowloon, Hong Kong \\ E-mail: mayangxq@polyu.edu.hk
}

April 26, 2006

\footnotetext{
${ }^{1}$ This research was partially supported by the Postdoctoral Fellowship Scheme of The Hong Kong Polytechnic University and the National Natural Science Foundation of China (Grant numbers: 60574073 and 10471142).

${ }^{2}$ Corresponding author.
} 


\begin{abstract}
In this paper, (weak) vector equilibrium principle with capacity constraints is introduced. A necessary condition that a vector minimum cost flow is a vector equilibrium flow with capacity constraints is obtained. When the number of paths connecting with each pair of source and sink is less than or equal to 2 , a sufficient condition for a vector minimum cost flow to be a vector equilibrium flow is also obtained. A generalized (weak) vector equilibrium principle is also introduced. Without any additional assumption, a necessary and sufficient condition for a (weak) vector minimum cost flow to be a generalized (weak) vector equilibrium flow is obtained.
\end{abstract}

Keywords: Traffic network equilibrium model, vector equilibrium principle, vector minimum cost flow. 


\section{Introduction}

The minimum cost flow problem on a network has extensively been investigated (see [1], [2] and [14]). The problem is to determine how some given amount of flows can be sent from some vertexes (the sources) to other vertexes (the sinks) at minimum cost, subject to the capacity limits on the arcs of the network. In general, the cost function of the problem is considered as a scalar one. In many practical situations, however, the choice of paths based on a single criterion by all retailers or manufacturers may not be reasonable. Minimum cost paths may be not ones of the least travel time. Naturally, some retailers or manufacturers may choose a freeway, of which tolls are collected, instead of the road in order that goods can be transported to a destination quickly. Of course, retailers or manufacturers do not choose the path which incurs both higher cost as well as longer delay than some other path. Therefore, a more realistic model is to take into account a vector cost function. The problem is called a vector minimum cost flow problem.

In 1993, a special issue for multiple objectives in transportation network design and routing was published in European Journal of Operations Research. In [5], Current and Marsh stated that methods for solving a vector minimum cost flow problem are classified as either being generating techniques or preference base techniques. Generating techniques are those which generate an exact representation or an approximation of the noninferior solution set. Preference based techniques educe preferences from the decision makers regarding the relative importance of the various objectives. These preferences are then incorporated into the mathematical formulation of the problem. In [9], Friesz et al. employed the 'weighting' method together with simulated annealing to generate the Pareto optimal set for continuous multiobjective optimal design of a transportation network. In [17], Tzeng and Chen obtained some noninferior solutions of traffic-assignment problem with three objectives by using the 'weighting' method and the Frank-Wolfe algorithm.

The earliest network equilibrium model was proposed by Wardrop for a transportation network. Since then, many other equilibrium models have also been proposed in the economics literature (see [7], [8], [10], [15] and [16]). Recently, 
equilibrium models based multicriteria consideration or vector-valued cost functions have been proposed (see [3], [4], [11]), [12] and [18]). In these papers, the classical Wardrop's principles without capacity constraints are generalized into weak vector equilibrium principle and vector equilibrium principle. Relationships between vector equilibrium principle and vector variational inequality and between vector equilibrium principle and a class of vector optimization problems were investigated.

We know that a minimum cost flow is equivalent to a class of equilibrium flows with capacity constraints under a linear scalar cost function with respect to arc flow (see Proposition 2.1). However, until now, there is no investigation on the vector equilibrium principle with upper and lower capacity constraints. There is also no discussion whether a vector minimum cost flow is a vector equilibrium flow with capacity constraints. In this paper, we first introduce a weak vector equilibrium principle and a vector equilibrium principle with capacity constraints, which are generalizations of the classic Wardrop's principle. We obtain a necessary condition for a vector minimum cost flow to be a vector equilibrium flow. When the number of paths connecting each pair of source and sink is less than or equal to 2, we obtain also a sufficient condition for a vector minimum cost flow to be a vector equilibrium flow. A counterexample is given to show that such a sufficient condition may not hold when there is a pair of source and sink such that the number of paths connecting them is greater than 2. We introduce new concepts of generalized weak vector equilibrium principle and generalized vector equilibrium principle. As such, we obtain a sufficient and necessary condition that a (weak) vector minimum cost flow is a generalized (weak) vector equilibrium flow without any additional assumption.

The outline of the paper is as follows. In Section 2, we introduce a vector minimum cost flow problem and a (weak) vector equilibrium principle. Then, we obtain a necessary condition for a vector minimum cost flow to be a vector equilibrium flow. We also illustrate that a vector minimum cost flow may not be a vector equilibrium flow. In Section 3, we introduce a generalized (weak) vector equilibrium principle and obtain a necessary and sufficient condition for a vector minimum cost flow to be a generalized vector equilibrium flow. 


\section{Vector minimum cost problem and vector equi- librium principle}

In this section, we consider a well-known equilibrium problem with capacity introduced on a transportation network with vector-valued cost functions. Our notation for the vector traffic network equilibrium follows closely that of Daniele and Maugeri [8]. Consider a transportation network $G=[\mathcal{N}, \mathcal{L}, L, U]$, where $\mathcal{N}$ denotes the set of nodes in the network, $\mathcal{L}$ is the set of directed arcs, $L$ and $U$ are the sets of lower and upper capacities of directed arcs, respectively. Let $a$ denote an arc of the network connecting a pair of nodes and $p$ denote a path, assumed to be acyclic, consisting of a sequence of arcs connecting an origin/destination (O/D) pair of nodes. Let $W$ be a set of $\mathrm{O} / \mathrm{D}$ pairs and $P_{w}$ denote the set of available paths joining the $\mathrm{O} / \mathrm{D}$ pair $w$. Let

$$
n=|\mathcal{L}|, \quad m=|W|, \quad \text { and } \quad P=\bigcup_{w \in W} P_{w} .
$$

Let $F_{p}$ denote the nonnegative flow on path $p$ and $F=\left\{F_{p}, \cdots, F_{m}\right\}^{T} \in \mathcal{R}^{m}$. The path flow vector $F$ induces a flow $f_{a}$ on arc $a \in \mathcal{L}$ given by:

$$
f_{a}=\sum_{p \in P} F_{p} \delta_{a p}, \quad \forall a
$$

where $\delta_{a p}=1$, if arc $a$ belongs to path $p$, and 0, otherwise. Hence, the load on an arc is equal to the sum of the flows of the paths that contain that arc. In addition, let $f=\left\{f_{a}, \cdots, f_{n}\right\}^{T}$ be the $n$-column vector of arc flow. Let $c_{a}\left(f_{a}\right): \mathcal{R} \rightarrow \mathcal{R}^{r}$ be a linear vector-valued cost function for $\operatorname{arc} a, C_{p}\left(F_{p}\right): \mathcal{R} \rightarrow \mathcal{R}^{r}$ be a linear vectorvalued cost function for path $p$ and the matrices $c(f)=\left(c_{a}\left(f_{a}\right), \cdots, c_{n}\left(f_{n}\right)\right) \in \mathcal{R}_{+}^{r \times n}$ and $C(F)=\left(C_{p}\left(F_{p}\right), \cdots, C_{m}\left(F_{m}\right)\right) \in \mathcal{R}_{+}^{r \times m}$. If the path costs are additive, then the vector-valued cost on the path is equal to the sum of the costs on the arcs composing it,

$$
C_{p}\left(F_{p}\right)=\sum_{a \in \mathcal{L}} \delta_{a p} c_{a}\left(f_{a}\right)
$$

We shall assume that the demand of traffic flow is fixed for each O/D pair $w$, i.e.,

$$
\sum_{p \in P_{w}} F_{p}=d_{w}, \quad \forall w
$$


where $d_{w} \in \mathcal{R}$ is a given demand for $\mathrm{O} / \mathrm{D}$ pair $w$, that is, the travel demand for an $\mathrm{O} / \mathrm{D}$ pair is equal to the sum of the flows on paths that join the O/D pair. For each path $p$, let

$$
\lambda_{p}=\max \left\{l_{a} \delta_{a p} \mid l_{a} \in L\right\}, \mu_{p}=\min \left\{u_{a} \delta_{a p} \mid u_{a} \in U\right\}
$$

and lower and upper capacity vectors

$$
\lambda=\left(\lambda_{p}, \cdots, \lambda_{m}\right)^{T} \text { and } \mu=\left(\mu_{p}, \cdots, \mu_{m}\right)^{T},
$$

respectively. For the $l$-dimensional Euclidean space $\mathcal{R}^{l}$, we denote the orderings induced by $\mathcal{R}_{+}^{l}$ as follows:

$$
\begin{array}{lll}
x \leq y & \text { iff } & y-x \in \mathcal{R}_{+}^{l} \\
x<y & \text { iff } & y-x \in \text { int } \mathcal{R}_{+}^{l},
\end{array}
$$

where $i n t \mathcal{R}_{+}^{l}$ is the interior of $\mathcal{R}_{+}^{l}$. The orderings $\geq$ and $>$ are defined similarly.

A flow $F \geq 0$ satisfying the demand requirements and capacity constraints is called a feasible flow, namely,

$$
\lambda \leq F \leq \mu
$$

and for every $w \in W$,

$$
\sum_{p \in P_{w}} F_{p}=d_{w}
$$

The set of feasible flow is given by

$$
\mathcal{K}=\left\{F \mid \lambda \leq F \leq \mu, \text { and } \sum_{p \in P_{w}} F_{p}=d_{w} \text {, for every } w \in W\right\},
$$

and it is called the feasible set. $\mathcal{K}$ is clearly a closed convex set.

The vector minimum cost flow problem with lower and upper capacity constrains and vector-valued cost functions is as follows:

$$
\begin{array}{ll}
(\mathrm{MCF}) \min \quad & \sum_{p \in P} C_{p}\left(F_{p}\right) \\
\text { s.t. } & \lambda \leq F \leq \mu, \\
& \sum_{p \in P_{w}} F_{p}=d_{w}, \quad \forall w \in W .
\end{array}
$$


Definition 2.1 (i) A flow $H \in \mathcal{K}$ of the problem $(M C F)$ is said to be a vector minimum cost flow if there does not exist any $F \in \mathcal{K}$ satisfying

$$
\sum_{p \in P} C_{p}\left(H_{p}\right)-\sum_{p \in P} C_{p}\left(F_{p}\right) \in \mathcal{R}_{+}^{l} \backslash\{0\}
$$

(ii) A flow $H \in \mathcal{K}$ of the problem $(M C F)$ is said to be a weak vector minimum cost flow if there does not exist any $F \in \mathcal{K}$ satisfying

$$
\sum_{p \in P} C_{p}\left(H_{p}\right)-\sum_{p \in P} C_{p}\left(F_{p}\right) \in i n t \mathcal{R}_{+}^{l}
$$

The well-known (scalar) Wardrop's user principle is a behavioral principle which asserts that when the traffic flow is in equilibrium, user only chooses minimum cost path to travel on. Chen and Yen [3] and Yang and Goh [18] generalized Wardrop's user principle and proposed vector equilibrium principle and weak vector equilibrium principle for vector equilibrium problem without lower and upper capacity constraints. Herein, we also propose two vector equilibrium principles for the vector traffic network equilibrium model with lower and upper capacity constraints.

Definition 2.2 (Vector equilibrium principle) $A$ flow $H \in \mathcal{K}$ is said to be in vector equilibrium if for all $O / D$ pairs $w$ and for any path $p, p^{\prime} \in P_{w}$, we have

$$
C_{p^{\prime}}-C_{p} \in \mathcal{R}_{+}^{l} \backslash\{0\} \Longrightarrow H_{p}=\mu_{p} \quad \text { or } \quad H_{p^{\prime}}=\lambda_{p^{\prime}} .
$$

Definition 2.3 (Weak vector equilibrium principle) $A$ flow $F \in \mathcal{K}$ is said to be in weak vector equilibrium if for all $O / D$ pairs $w$ and for any path $p, p^{\prime} \in P_{w}$, we have

$$
C_{p^{\prime}}-C_{p} \in i n t \mathcal{R}_{+}^{l} \Longrightarrow H_{p}=\mu_{p} \quad \text { or } \quad H_{p^{\prime}}=\lambda_{p^{\prime}}
$$

Theorem 2.1 If a flow $H \in \mathcal{K}$ is a vector minimum cost flow of the problem $(M C F)$, then the flow $H$ is a vector equilibrium flow.

Proof. Suppose that $H$ is not a vector equilibrium flow. Then, there exist $w \in W$ and $q, s \in P_{w}$ such that

$$
C_{s}-C_{q} \in \mathcal{R}_{+}^{l} \backslash\{0\} \Longrightarrow H_{q}<\mu_{q} \text { and } H_{s}>\lambda_{s} .
$$


Construct a flow $F$ as follows:

$$
F_{r}= \begin{cases}H_{r}, & r \neq q, s \\ H_{q}+\varepsilon, & r=q, \\ H_{s}-\varepsilon, & r=s,\end{cases}
$$

where

$$
0<\varepsilon \leq \min \left\{\mu_{q}-H_{q}, H_{s}-\lambda_{s}\right\}
$$

Then, we have

$$
\lambda \leq F \leq \mu
$$

and

$$
\sum_{p \in P_{w}} F_{p}=d_{w}, \quad \forall w \in W
$$

i.e.,

$$
F \in \mathcal{K} .
$$

It follows readily that

$$
\begin{aligned}
\sum_{p \in P} C_{p}\left(F_{p}\right)-\sum_{p \in P} C_{p}\left(H_{p}\right) & =C_{q}\left(H_{q}+\varepsilon\right)-C_{q}\left(H_{q}\right)+C_{s}\left(H_{s}-\varepsilon\right)-C_{s}\left(H_{s}\right) \\
& =C_{q}(\varepsilon)-C_{s}(\varepsilon) \\
& =\left(C_{q}-C_{s}\right)(\varepsilon) \in-\mathcal{R}_{+}^{l} \backslash\{0\},
\end{aligned}
$$

which contradicts to the assumption condition that the flow $H$ is a vector minimum cost flow of the problem $(M C F)$.

Theorem 2.2 If a flow $H \in \mathcal{K}$ is a vector equilibrium flow and $\left|P_{w}\right| \leq 2, \forall w \in W$, then the flow $H$ is a vector minimum cost flow of $(M C F)$.

Proof. Take any $w \in W$. We shall prove that the flow $\tilde{H}_{w}=\left\{H_{p} \mid p \in P_{w}\right\}$ is a vector minimum cost flow of the following problem:

$$
\begin{aligned}
\left(M C F_{w}\right) \quad \min & \sum_{p \in P_{w}} C_{p}\left(F_{p}\right) \\
\text { s.t. } & \lambda_{p} \leq F_{p} \leq \mu_{p}, \text { for } p \in P_{w} \\
& \sum_{p \in P_{w}} F_{p}=d_{w} .
\end{aligned}
$$


In fact, if $\left|P_{w}\right|=1$, the flow $\tilde{H}_{w}$ is a vector minimum cost flow of the problem $\left(M C F_{w}\right)$ naturally.

If $\left|P_{w}\right|=2$ and the flow $\tilde{H}_{w}$ is not a vector minimum cost flow of the problem $\left(M C F_{w}\right)$, there exists a feasible flow $\tilde{F}_{w}$ of the problem $\left(M C F_{w}\right)$ such that

$$
\sum_{p \in P_{w}} C_{p}\left(\tilde{F}_{p}\right)-\sum_{p \in P_{w}} C_{p}\left(H_{p}\right) \in-\mathcal{R}_{+}^{l} \backslash\{0\}
$$

i.e.,

$$
\sum_{p \in P_{w}} C_{p}\left(\tilde{F}_{p}-H_{p}\right) \in-\mathcal{R}_{+}^{l} \backslash\{0\} .
$$

Let $P_{w}=\left\{p_{1}, p_{2}\right\}$. Since $\tilde{F}_{w}$ and $\tilde{H}_{w}$ are feasible flows of $\left(M C F_{w}\right)$,

$$
F_{p_{1}}+F_{p_{2}}=H_{p_{1}}+H_{p_{2}}=d_{w}
$$

Then, by (4) and (5) we have

$$
\left(C_{p_{1}}-C_{p_{2}}\right)\left(F_{p_{1}}-H_{p_{1}}\right) \in-\mathcal{R}_{+}^{l} \backslash\{0\}
$$

It follows from $(6)$ and $H \neq F$ that one of the following two cases happens:

1. If $F_{p_{1}}-H_{p_{1}}>0, C_{p_{1}}-C_{p_{2}} \in-\mathcal{R}_{+}^{l} \backslash\{0\}$;

2. If $F_{p_{1}}-H_{p_{1}}<0, C_{p_{1}}-C_{p_{2}} \in \mathcal{R}_{+}^{l} \backslash\{0\}$.

We can see that any one of the two cases contradicts to the definition of vector equilibrium flow (1). Thus, the flow $\tilde{H}_{w}$ is a vector minimum cost flow of the problem $\left(M C F_{w}\right)$. Naturally, the flow $H$ is a vector minimum cost flow of the problem $(M C F)$. The proof of the result is complete.

Following the proofs of Theorems 2.1 and 2.2, we can establish two similar results between weak vector minimum cost flow and weak vector equilibrium flow.

Theorem 2.3 If a flow $H \in \mathcal{K}$ is a weak vector minimum cost flow of the problem $(M C F)$, then the flow $H$ is a weak vector equilibrium flow.

Theorem 2.4 If a flow $H \in \mathcal{K}$ is a weak vector equilibrium flow and $\left|P_{w}\right| \leq 2, \forall w \in$ $W$, then the flow $H$ is a weak vector minimum cost flow of (MCF). 
Remark 2.1 If there is a $w \in W$ such that $\left|P_{w}\right|>2$, Theorem 2.2 may not hold. The following example is given to explain the situation.

Example 2.1 Given a transportation network depicted in Figure 1, which consists of two nodes $x$ and $y$, three $\operatorname{arcs} a, b$ and $d$ and a single O/D pair $w=(x, y)$.

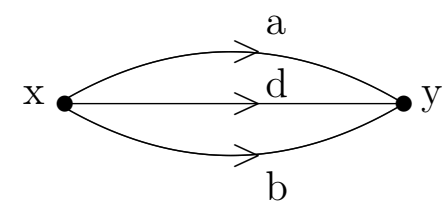

Figure 1. Network topology for an example with $\left|P_{w}\right|>2$.

Assume that upper capacities of the three $\operatorname{arcs} a, b$ and $d$ are 4,3 and 3 , respectively, and their lower capacities are all zero. A conventional traffic-assignment problem in Figure 1 is to simulate or predict the trip-flow pattern between the origin $x$ and the destination $y$ under the conditions that satisfy upper and lower constraints. Suppose that the basic model consists of two objective, which are travel cost and travel time. Like [17], the model can be expressed as follows.

$$
\min \left(g_{1}, g_{2}\right)
$$

subject to

$$
\begin{aligned}
& \lambda \leq F \leq \mu, \\
& \sum_{p \in P_{w}} F_{p}=d_{w}, \\
& g_{1}=f_{a} \cdot d_{a}+f_{b} \cdot d_{b}+f_{d} \cdot d_{d}, \\
& g_{2}=f_{a} \cdot t_{a}+f_{b} \cdot t_{b}+f_{d} \cdot t_{d},
\end{aligned}
$$

where

$g_{1}-$ total travel cost,

$g_{2}$ - total travel time,

$d_{\alpha}-$ travel cost of arc $\alpha$,

$t_{\alpha}$ - travel time of arc $\alpha$, 
$d_{w}$ - travel demand for $w$

$f_{\alpha}-$ flow of arc $\alpha$,

$F_{p}$ - flow of the path $p$ from origin $x$ to destination $y$,

$\lambda=\left(\begin{array}{l}0 \\ 0 \\ 0\end{array}\right), \mu=\left(\begin{array}{l}4 \\ 3 \\ 3\end{array}\right), F=\left(\begin{array}{c}F_{a} \\ F_{b} \\ F_{d}\end{array}\right), W=\{w=(x, y)\}$, and $P_{w}=P=\{a, b, d\}$.

Furthermore, suppose that $d_{w}=4$,

$$
c_{a}=\left(\begin{array}{c}
d_{a} \\
t_{a}
\end{array}\right)=\left(\begin{array}{l}
6 \\
3
\end{array}\right), \quad c_{b}=\left(\begin{array}{c}
d_{a} \\
t_{a}
\end{array}\right)=\left(\begin{array}{l}
5 \\
4
\end{array}\right) \quad \text { and } \quad c_{d}=\left(\begin{array}{c}
d_{a} \\
t_{a}
\end{array}\right)=\left(\begin{array}{l}
7 \\
1
\end{array}\right)
$$

Then, we have

$$
\begin{gathered}
C_{a}=c_{a}, \quad C_{b}=c_{a}, \quad C_{d}=c_{d}, \\
F_{a}=f_{a}, F_{b}=f_{b}, \text { and } F_{d}=f_{d} .
\end{gathered}
$$

Thus, we can transform the traffic-assignment problem into the following vector minimum cost flow problem (MCF):

$$
\begin{array}{ll}
\min & \sum_{p \in P} C_{p}\left(F_{p}\right) \\
\text { s.t. } & \lambda \leq F \leq \mu, \\
& \sum_{p \in P_{w}} F_{p}=d_{w}, \quad \forall w \in W .
\end{array}
$$

It follows readily that any feasible flow is a vector equilibrium flow. Take $H_{a}=$ 4, $H_{b}=0$ and $H_{d}=0$. It is a feasible flow. Thus, $H$ is a vector equilibrium flow. However, take $F_{a}=0, F_{b}=2$ and $F_{d}=2$. We have that $F$ is a feasible flow and

$$
\begin{aligned}
\sum_{p \in P} C_{p}\left(F_{p}\right)-\sum_{p \in P} C_{p}\left(H_{p}\right) & =c_{a}\left(F_{a}-H_{a}\right)+c_{b}\left(F_{b}-H_{b}\right)+c_{d}\left(F_{d}-H_{d}\right) \\
& =-c_{a}\left(H_{a}\right)+c_{b}\left(F_{b}\right)+c_{d}\left(F_{d}\right) \\
& =\left(\begin{array}{c}
0 \\
-2
\end{array}\right) \in-\mathcal{R}_{+}^{l} \backslash\{0\}
\end{aligned}
$$

Thus, the vector equilibrium flow $H$ is not a vector minimum cost flow. 
Remark 2.2 It follows from Theorems 2.1 and 2.2 and Example 2.1 that a vector minimum cost flow of the problem $(M C F)$ is a vector equilibrium flow and a vector equilibrium flow is, in general, not a vector minimum cost flow of the problem $(M C F)$ when there exists a $w \in W$ such that $\left|P_{w}\right|>2$. However, if cost functions of a traffic network problem are scalar ones, then a minimum cost flow is equivalent to an equilibrium flow. The following proposition states the result:

Proposition 2.1 If for each $p \in P, C_{p}$ is a single-valued function from $\mathcal{R}$ to $\mathcal{R}$, then a flow $H \in \mathcal{K}$ is a minimum cost flow of the problem $(M C F)$ if and only if the flow $H \in \mathcal{K}$ is an equilibrium flow.

ProOF. It follows from Theorem 2.1 that we only need to prove the sufficient property. We shall use the method of contradiction to prove it. Suppose that there is a flow $F \in \mathcal{K}$ such that

$$
\sum_{p \in P} C_{p}\left(F_{p}\right)<\sum_{p \in P} C_{p}\left(H_{p}\right)
$$

i.e.,

$$
\sum_{p \in P} C_{p}\left(F_{p}-H_{p}\right)<0
$$

Since $F, H \in \mathcal{K}$ and $F \neq H$, there exist two index sets $I_{1}$ and $I_{2}$ of paths, where $\left|I_{1}\right|+\left|I_{2}\right| \leq|P|$, such that

$$
\begin{gathered}
F_{p}-H_{p}>0, \forall p \in I_{1}, \\
F_{q}-H_{q}<0, \forall q \in I_{2} \\
F_{s}-H_{s}=0, \forall s \in P \backslash\left(I_{1} \bigcup I_{2}\right),
\end{gathered}
$$

and

$$
\sum_{p \in I_{1}}\left(F_{p}-H_{p}\right)=\sum_{q \in I_{2}}\left(H_{q}-F_{q}\right)
$$

If for any $p \in I_{1}$ and $q \in I_{2}$,

$$
C_{p} \geq C_{q}
$$

then we have

$$
\sum_{p \in P} C_{p}\left(H_{p}\right) \leq \sum_{p \in P} C_{p}\left(F_{p}\right)
$$


which contradicts to (7). Therefore, there exist a $p \in I_{1}$ and a $q \in I_{2}$ such that

$$
C_{p}<C_{q}
$$

Since $H$ is an equilibrium flow,

$$
C_{p}<C_{q} \Longrightarrow H_{p}=\mu_{p} \text { or } H_{q}=\lambda_{q}
$$

which contradicts to (8) or (9).

Remark 2.3 In Lemma 2.1 of [6], Daniele et al also introduced a statement to be the same as Definition 2.3 in the scalar case and investigated an equivalent relationship between the statement and a class of variational inequality problems. The solution for the class of variational inequality problems, in fact, is equivalent to the minimum cost flow of the problem $(M C F)$ in scalar case. Thus, Proposition 2.1 can be considered as a corollary of Lemma 2.1 in [6]. Here, we only gave an alternative method to prove it.

\section{Generalized vector equilibrium principle}

It follows from Theorems 2.1 and 2.2 and Example 2.1 that the vector equilibrium principle introduced similar to the well-known (scalar) Wardrop's user principle does not have all the properties of a vector minimum cost flow. We need to introduce new concepts on generalized vector equilibrium flows. On this thesis, we will establish the equivalence relationship between vector minimum cost flows and generalized vector equilibrium flows. These new concepts are given in the following definitions.

Definition 3.1 (Generalized vector equilibrium principle) $A$ flow $F \in \mathcal{K}$ is said to be in generalized vector equilibrium if for all $O / D$ pairs $w$ and for any integer numbers $l_{1}, l_{2}>0$ and $l_{1}+l_{2} \leq\left|P_{w}\right|$, we have

$$
\begin{aligned}
\sum_{j=1}^{l_{2}} \theta_{j} C_{p_{j}^{\prime}}-\sum_{i=1}^{l_{1}} \eta_{i} C_{p_{i}} \in \mathcal{R}_{+}^{l} \backslash\{0\} \Longrightarrow \quad & \exists i_{0}\left(0<i_{0} \leq l_{1}\right) \text { s.t. } H_{p_{i_{0}}}=\mu_{p_{i_{0}}} \text { or } \\
& \exists j_{0}\left(0<j_{0} \leq l_{2}\right) \text { s.t. } H_{p_{j_{0}}^{\prime}}=\lambda_{p_{j_{0}}^{\prime}},
\end{aligned}
$$


where $p_{i}, p_{j}^{\prime} \in P_{w}, \eta_{i}, \theta_{j}>0, i=1, \cdots, l_{1}, j=1, \cdots, l_{2}$,

$$
\sum_{i=1}^{l_{1}} \eta_{i}=1 \text { and } \sum_{j=1}^{l_{2}} \theta_{j}=1 .
$$

Definition 3.2 (Generalized weak vector equilibrium principle) $A$ flow $F \in \mathcal{K}$ is said to be in generalized weak vector equilibrium if for all $O / D$ pairs $w$ and for any $l_{1}, l_{2}>0$ and $l_{1}+l_{2} \leq\left|P_{w}\right|$, we have

$$
\begin{aligned}
\sum_{i=1}^{l_{1}} \eta_{i} C_{p_{i}}<\sum_{j=1}^{l_{2}} \theta_{j} C_{p_{j}^{\prime}} \Longrightarrow \quad & \exists i_{0}\left(0<i_{0} \leq l_{1}\right) \text { s.t. } H_{p_{i_{0}}}=\mu_{p_{i_{0}}} \text { or } \\
& \exists j_{0}\left(0<j_{0} \leq l_{2}\right) \text { s.t. } H_{p_{j_{0}}^{\prime}}=\lambda_{p_{j_{0}}^{\prime}},
\end{aligned}
$$

where $p_{i}, p_{j}^{\prime} \in P_{w}, \eta_{i}, \theta_{j}>0, i=1, \cdots, l_{1}, j=1, \cdots, l_{2}$,

$$
\sum_{i=1}^{l_{1}} \eta_{i}=1 \text { and } \sum_{j=1}^{l_{2}} \theta_{j}=1 .
$$

Definition 3.2 means that if there is a set of paths such that the nonzero convex combination of vector cost functions on the paths in this set is less than the nonzero convex combination of vector cost functions on another set of paths, then either there exists a path $p_{i_{0}}$ in the former set such that the flow on the path $p_{i_{0}}$ is equal to the upper capacity of the path $p_{i_{0}}$ or there exists a path $p_{j_{0}}^{\prime}$ in the latter set such that the flow on the path $p_{j_{0}}^{\prime}$ is equal to the lower capacity of the path $p_{j_{0}}^{\prime}$. Naturally, we may also use same way to understand the meaning of Definition 3.1.

Remark 3.1 When $l_{1}=1$ and $l_{2}=1$, the cases described in Definitions 3.1 and 3.2 are, in fact, the vector equilibrium principle and the weak vector equilibrium principle, respectively. Thus, the generalized vector equilibrium flow in Definition 3.1 and the generalized weak vector equilibrium flow in Definition 3.2 are stronger than the vector equilibrium flow in Definition 2.2 and the weak vector equilibrium flow in Definition 2.3, respectively.

Remark 3.2 When for each $p \in P, C_{p}$ is a single-valued function from $\mathcal{R}$ to $\mathcal{R}$, a flow satisfies the generalized vector equilibrium principle if and only if it satisfies the vector equilibrium principle. 
Theorem 3.1 A flow $H \in \mathcal{K}$ is a vector minimum cost flow of the problem $(M C F)$ if and only if the flow $H \in \mathcal{K}$ is a generalized vector equilibrium flow.

Proof. Suppose that the flow $H \in \mathcal{K}$ is a vector minimum cost flow and is not a generalized vector equilibrium flow. Then there exist a $\mathrm{O} / \mathrm{D}$ pair $w, l_{1}, l_{2}, \eta_{i}>$ $0, \sum_{i=1}^{l_{1}} \eta_{i}=1, i=1, \cdots, l_{1}$ and $\theta_{j}>0, \sum_{j=1}^{l_{2}} \theta_{j}=1, j=1, \cdots, l_{2}$ such that

$$
\sum_{i=1}^{l_{1}} \eta_{i} C_{p_{i}}-\sum^{l_{2}} \theta_{j} C_{p_{j}^{\prime}} \in-\mathcal{R}_{+}^{l} \backslash\{0\}
$$

and

$$
H_{p_{i}}<\mu_{p_{i}}, \quad i=1, \cdots, l_{1} \text { and } H_{p_{j}}>\lambda_{p_{j}}, \quad j=1, \cdots, l_{2} .
$$

Construct a flow as follows:

$$
F_{r}= \begin{cases}H_{r}, & r \neq p_{i}, p_{j}^{\prime}, i=1, \cdots, l_{1}, j=1, \cdots, l_{2}, \\ H_{p_{i}}+\eta_{i} \varepsilon, & r=p_{i}, i=1, \cdots, l_{1}, \\ H_{p_{j}^{\prime}}-\theta_{j} \varepsilon, & r=p_{j}^{\prime}, j=1, \cdots, l_{2},\end{cases}
$$

where

$$
0<\varepsilon \leq \min _{i=1, \cdots, l_{1}, j=1, \cdots, l_{2}}\left\{\mu_{p_{i}}-H_{p_{i}}, H_{p_{j}^{\prime}}-\lambda_{p_{j}^{\prime}}\right\}
$$

Then, we have

$$
\lambda \leq F \leq \mu
$$

and

$$
\sum_{p \in P_{w}} F_{p}=d_{w}, \quad \forall w \in W
$$

i.e.,

$$
F \in \mathcal{K}
$$

It follows readily that

$$
\begin{aligned}
\sum_{p \in P} C_{p}\left(F_{p}\right)-\sum_{p \in P} C_{p}\left(H_{p}\right)= & \sum_{i=1}^{l_{1}}\left(C_{p_{i}}\left(H_{p_{i}}+\eta_{i} \varepsilon\right)-C_{p_{i}}\left(H_{p_{i}}\right)\right)+ \\
& \sum_{j=1}^{l_{2}}\left(C_{p_{j}^{\prime}}\left(H_{p_{j}^{\prime}}-\theta_{j} \varepsilon\right)-C_{p_{j}^{\prime}}\left(H_{p_{j}^{\prime}}\right)\right)
\end{aligned}
$$




$$
\begin{aligned}
& =\sum_{i=1}^{l_{1}} C_{p_{i}}\left(\eta_{i} \varepsilon\right)-\sum_{j=1}^{l_{2}} C_{p_{j}^{\prime}}\left(\theta_{j} \varepsilon\right) \\
& =\left(\sum_{i=1}^{l_{1}} \eta_{i} C_{p_{i}}-\sum_{j=1}^{l_{2}} \theta_{j} C_{p_{j}^{\prime}}\right)(\varepsilon) \in-\mathcal{R}_{+}^{l} \backslash\{0\},
\end{aligned}
$$

which contradicts to the assumption condition that the flow $H$ is a vector minimum cost flow of the problem $(M C F)$.

Conversely, suppose that the flow $H \in \mathcal{K}$ is a vector equilibrium flow. We shall prove that for any $w \in W$, the flow $\tilde{H}_{w}=\left\{H_{p} \mid p \in P_{w}\right\}$ is a vector minimum cost flow of the following problem:

$$
\begin{aligned}
\left(M C F_{w}\right) \quad \min & \sum_{p \in P_{w}} C_{p}\left(F_{p}\right) \\
\text { s.t. } & \lambda_{p} \leq F_{p} \leq \mu_{p}, \text { for } p \in P_{w} \\
& \sum_{p \in P_{w}} F_{p}=d_{w} .
\end{aligned}
$$

In fact, if the flow $\tilde{H}_{w}$ is not a vector minimum cost flow of the problem $\left(M C F_{w}\right)$, there exists a feasible flow $\tilde{F}_{w}=\left\{F_{p} \mid p \in P_{w}\right\}$ of the problem $\left(M C F_{w}\right)$ such that

$$
\sum_{p \in P_{w}} C_{p}\left(F_{p}\right)-\sum_{p \in P_{w}} C_{p}\left(H_{p}\right) \in-\mathcal{R}_{+}^{l} \backslash\{0\}
$$

i.e.,

$$
\sum_{p \in P_{w}} C_{p}\left(F_{p}-H_{p}\right) \in-\mathcal{R}_{+}^{l} \backslash\{0\} .
$$

Since the flows $\tilde{F}_{w}$ and $\tilde{H}_{w}$ are two feasible flows of the problem $\left(M C F_{w}\right)$ and $\tilde{F}_{w} \neq$ $\tilde{H}_{w}$, there exist $l_{1}, l_{2}>0, l_{1}+l_{2} \leq\left|P_{w}\right|$ and $p_{i}, p_{j}^{\prime} \in P_{w}, i=1, \cdots, l_{1}, j=1, \cdots, l_{2}$ such that

$$
\begin{aligned}
& F_{p_{i}}-H_{p_{i}}>0, i=1, \cdots, l_{1}, \\
& F_{p_{j}^{\prime}}-H_{p_{j}^{\prime}}<0, j=1, \cdots, l_{2},
\end{aligned}
$$

and

$$
F_{p}-H_{p}=0, p \in P_{w} \backslash\left(\left\{p_{i} \mid i=1, \cdots, l_{1}\right\} \bigcup\left\{p_{j}^{\prime} \mid j=1, \cdots, l_{2}\right\}\right) .
$$

By the feasibility of $\tilde{F}_{w}$ and $\tilde{H}_{w}$, we have

$$
\sum_{i=1}^{l_{1}}\left(F_{p_{i}}-H_{p_{i}}\right)=\sum_{j=1}^{l_{2}}\left(H_{p_{j}^{\prime}}-F_{p_{j}^{\prime}}\right) .
$$


Set

$$
\eta_{i}=\frac{F_{p_{i}}-H_{p_{i}}}{\sum_{i=1}^{l_{1}}\left(F_{p_{i}}-H_{p_{i}}\right)} \text { and } \theta_{j}=\frac{H_{p_{j}^{\prime}}-F_{p_{j}^{\prime}}}{\sum_{j=1}^{l_{2}}\left(H_{p_{j}^{\prime}}-F_{p_{j}^{\prime}}\right)} .
$$

Then, $0<\eta_{i}, \theta_{j}, i=1, \cdots, l_{1}, j=1, \cdots, l_{2}$ and

$$
\sum_{i=1}^{l_{1}} \eta_{i}=1 \text { and } \sum_{j=1}^{l_{2}} \theta_{j}=1
$$

It follows from (10) that

$$
\sum_{i=1}^{l_{1}} \eta_{i} C_{p_{i}}-\sum_{j=1}^{l_{2}} \theta_{j} C_{p_{j}^{\prime}} \in-\mathcal{R}_{+}^{l} \backslash\{0\} .
$$

By Definition 3.1, there exists $0<i_{0} \leq l_{1}$ such that

$$
H_{p_{i_{0}}}=\mu_{p_{i_{0}}}
$$

or there exists $0<j_{0} \leq l_{2}$ such that

$$
H_{p_{j_{0}}}=\lambda_{p_{j_{0}}}
$$

which contradicts to $(11)$ or $(12)$. Thus, the flow $\tilde{H}_{w}$ is a vector minimum cost flow of the problem $\left(M C F_{w}\right)$. Naturally, the flow $H$ is a vector minimum cost flow of the problem $(M C F)$.

Example 3.1 In this example, we shall continue to consider Example 2.1. We shall use Theorem 3.1 to explain that the feasible flow $H$ in Example 2.1 is not a vector minimum cost flow. Take $l_{1}=2, \eta_{1}=\eta_{2}=\frac{1}{2}$ and $l_{2}=1, \theta_{1}=1$. We have

$$
\begin{aligned}
\theta_{1} C_{a}-\left(\eta_{1} C_{b}+\eta_{2} C_{d}\right) & =\left(\begin{array}{l}
6 \\
3
\end{array}\right)-\left(\frac{1}{2}\left(\begin{array}{l}
5 \\
4
\end{array}\right)+\frac{1}{2}\left(\begin{array}{l}
7 \\
1
\end{array}\right)\right) \\
& =\left(\begin{array}{l}
0 \\
0.5
\end{array}\right) \in \mathcal{R}_{+}^{2} \backslash\{0\} .
\end{aligned}
$$

It follows from Example 2.1 that

$$
H_{a}=4, H_{b}=0 \text { and } H_{d}=0 .
$$

Obviously, the flow of $H$ on the path $a$ is not equal to the lower capacity of the path and there is not a path in paths $b$ and $d$ such that the flow of $H$ on the 
path is equal to its upper capacity. Thus, the feasible flow $H$ does not satisfy the generalized vector equilibrium principle. From Theorem 3.1, the flow $H$ is not a vector minimum cost flow.

Following the proof of Theorem 3.1, we can obtain a similar result between weak minimum cost flow and weak vector equilibrium flow.

Theorem 3.2 A flow $H \in \mathcal{K}$ is a weak vector minimum cost flow of the problem $(M C F)$ if and only if the flow $H \in \mathcal{K}$ is a generalized weak vector equilibrium flow.

\section{Conclusions}

In this paper, we first introduced the (weak) vector equilibrium principle with capacity constraints and obtained a necessary condition for a vector minimum cost flow to be a vector equilibrium flow. We also introduced generalized (weak) vector equilibrium principle. By using this concept, we proved a sufficient and necessary condition that a (weak) vector minimum cost flow is a generalized (weak) vector equilibrium flow in a transportation network with upper and lower capacity constraints.

\section{References}

[1] Ahuja, R. K., Magnanti, T. L. and Orlin, J. B., Network flows: theory, algorithms and applications, Prentice-Hall, Englewood Cliffs, NJ, 1993.

[2] Assad, A.A., Multicommodity network flows: a survey, Networks 8 (1978)37-91.

[3] Chen, G.Y. and Yen, N.D., On the variational inequality model for network equilibrium, Internal Report 3.196 (724), Department of Mathematics, University of Pisa, 1993.

[4] Chen, G. Y., Goh, C. J. and Yang, X. Q., Vector network equilibrium problems and nonlinear scalarization methods, Mathematical Methods of Operations Research 49 (1999)239-253. 
[5] Current, J. and Marsh, M., Multiobjective transportation network design and routing problems: Taxonomy and annotation, European Journal of Operations Research, 65(1993)4-19.

[6] Daniele, P., Maugeri, A. and Oettli, W., Time-Dependent Traffic Equilibria, Journal of Optimization Theory and Applications 103 (1999)543-555.

[7] Daniele, P., Franco Giannessi and Antonino Maugeri, Equilibrium problems and variational models. Including papers from the meeting held in Erice, June 23-July 2, 2000, Nonconvex Optimization and its Applications, 68. Kluwer Academic Publishers, Norwell, MA, 2003.

[8] Daniele, P. and Maugeri, A., Variational inequalities and discrete and continuum models of network equilibrium problems, Mathematical and Computer Modeling, 35 (2000)393-411.

[9] Friesz, T. L., Anandalingam, G., Mehta, N. J., Nam, K., Shah, S. J. and Tobin, R. L., The multiobjective equilibrium network design problem revisited: A simulated annealing approach, European Journal of Operations Research, 65(1993)44-57.

[10] Giannessi, F. and Maugeri, A., Variational inequalities and network equilibrium problems. Proceedings of the conference held in Erice, June 19-25, 1994, Plenum Press, New York, 1995.

[11] Giannessi, F., Vector variational inequalities and vector equilibria, Kluwer Academic Publisher 2000.

[12] Goh, C.J. and Yang, X.Q, Theory and methodology of vector equilibrium problem and vector optimization, European Journal of Operational Research 116 (1999)615-628.

[13] Kinderlehrer, D. and Stampacchia, G., An introduction to variational inequalities and their applications Academic Press, 1980.

[14] Maurras, J. F. and Vaxes, Y., Multicommodity network flow with jump constraints, Discrete Mathematics 165/166 (1997)481-486. 
[15] Nagurney, A., Network economics, A variational inequality approach, Kluwer Academic Publishers, Dordrechit, 1999.

[16] Nagurney, A. and Dong, J., Supernetworks decision-making for the information age, Edward Elgar, Cheltenham, UK • Northampton, MA, USA, 2002.

[17] Tzeng, G. H. and Chen, C. H., Multiobjective decision making for traffic assignment, IEEE Transactyions of Engineering Management, 40(1993)180-187.

[18] Yang, X. Q. and Goh, C. J., On vector variational inequalities: application to vector equilibria, Journal of Optimization Theory and Applications 95 (1997)431-443. 